\title{
Life Quality and Cytokines Profile in Patients with Asthma and Osteoarthritis
}

\author{
Yuliya S. Ivanchuk, PGS; Ludmila V. Tribuntceva, PhD; Andrey V. Budnevsky, PhD, ScD; \\ Yanina S. Shkatova, PGS; Evgeniy S. Ovsyannikov, PhD, ScD*; Roman E. Tokmachev, PhD \\ Voronezh State Medical University named after N. N. Burdenko \\ Voronezh, the Russian Federation
}

\begin{abstract}
The objective of this study was to evaluate levels of leptin, adiponectin, IL-4, IL-6, TNF- $\alpha$, oxidative damage, and antioxidant status in patients with bronchial asthma (BA), compared to patients who suffer from both BA and osteoarthritis (OA), and analyze the quality of life in such patients.

Methods and Results: The study included 103 patients (34 men and 69 women) diagnosed with moderate asthma aged from 30 to 70 years (mean age of 58.52 \pm .14 years). The levels of IL-4, IL-6, TNF- $\alpha$, adiponectin, leptin, total antioxidant status (TAS), and total oxidative damage (TOD) were measured. Two questionnaires were used in this study: Asthma Quality of Life Questionnaire (AQLQ) and Asthma Control Test (ACT).

The levels of leptin, TNF- $\alpha$, and Il- 6 were significantly higher in Group 2 than in Group 1. On the contrary, the IL-4 level was higher in Group 1 than in Group 2. The TAS value was significantly higher in Group 1 than in Group $2(P=0.0001)$. The TOD value was significantly higher in Group 2 than in Group $1(P=0.0000)$. The domains of AQLQ(S) activity, symptoms, and emotions were decreased in patients of Group 2. The values of the ACT test were 18.0 2.61 points and $16.78 \pm 1.92$ points in Group 1 and Group 2, respectively $(P=0.0077)$.

Conclusion: In patients with both asthma and osteoarthritis, levels of inflammatory cytokines, such as leptin, IL-6, and TNF- $\alpha$, are significantly elevated as well as values of total oxidative status, which correlate with poorer asthma control and quality of life.(International Journal of Biomedicine. 2021;11(2):137-140.)
\end{abstract}

Key Words: asthma $\bullet$ osteoarthritis $\bullet \mathrm{IL}-6 \cdot \mathrm{TNF}-\alpha \cdot$ quality of life

For citation: Ivanchuk YuS, Tribuntceva LV, Budnevsky AV, Shkatova YaS, Ovsyannikov ES, Tokmachev RE. Life Quality and Cytokines Profile in Patients with Asthma and Osteoarthritis. International Journal of Biomedicine. 2021;11(2):137-140. doi:10.21103/Article11(2)_OA2

\section{Abbreviations}

OA, osteoarthritis; BMI, body mass index; BA, bronchial asthma; IL, interleukin; TNF-a, tumor necrosis factor alpha; TAS, total antioxidant status; TOD, total oxidative damage.

\section{Introduction}

Among respiratory diseases, bronchial asthma (BA) is one of the most common; almost 250,000 patients die from BA every year as a result of severe exacerbation. ${ }^{(1)}$ Asthma affects more than 300 million people in the world and costs over $\$ 80$ billion annually in the United States. ${ }^{(2)}$ It has been shown that asthmatic patients often have comorbidities, which influence their overall health, life quality, and clinical course of asthma. ${ }^{(3,4)}$ The most researched comorbidities of asthma include cardiovascular diseases, cerebrovascular diseases, mental diseases, diabetes mellitus, and obesity. ${ }^{(5-8)}$ Osteoarthritis (OA) is a less studied comorbidity; however, according to a German telephone interview health survey, German Health Update (GEDA) 2009 and 2010 , OA was noted in $18.4 \%$ of all patients with no current asthma and in $29.4 \%$ of patients with current asthma, making this comorbidity one of the most common ones, following closely after cardiovascular diseases. ${ }^{(9)}$ Nevertheless, the data is 
limited on the association between BA and OA, as well as on the influence these diseases might have on each other.

The objective of this study was to evaluate levels of leptin, adiponectin, IL-4, IL- 6 , TNF- $\alpha$, oxidative damage, and antioxidant status in patients with BA, compared to patients who suffer from both BA and OA, and analyze the quality of life in such patients.

\section{Materials and Methods}

The study included 103 patients ( 34 men and 69 women) diagnosed with moderate asthma aged from 30 to 70 years (mean age of 58.52 \pm 7.14 years). Excluded criteria were mental illness, rheumatic diseases, fibromyalgia, tuberculosis, severe and decompensated diseases of liver and kidneys, severe and decompensated cardiovascular diseases, cancer, pregnancy and lactation, severe infectious diseases, and previous joint surgery.

The study was approved by the Ethics Committee of Voronezh State Medical University named after N.N. Burdenko. Written informed consent was obtained from each patient.

The diagnosis of asthma was made according to GINA. We analyzed complaints, anamnesis data, objective status data, and laboratory and instrumental data (spirometry with a 400-mg salbutamol test). All patients received standard asthma therapy.

Two questionnaires were used in this study: Asthma Quality of Life Questionnaire (AQLQ) and Asthma Control Test (ACT).

The levels of IL-4, IL-6, TNF- $\alpha$, adiponectin, leptin, TAS, and TOD were measured. The levels of leptin and adiponectin were measured using the appropriate reagent kits for quantitative determination of leptin and adiponectin in serum. The determination of TOD was carried out with use of a reagent kit to determine the degree of TOD to biological molecules (PerOx (TOS) (Oxidative Capacity)). We determined the overall antioxidant status by using reagents for determining TAS (ImAnOx (TAS) (Antioxidative Capacity)). The IL-4 level was determined by using a set of reagents for the EIA test; the IL-6 level was determined by using a set of reagents for the ELISA test.

All data was evaluated with STATGRAPHICS Plus 5.1. Baseline characteristics were summarized as frequencies and percentages for categorical variables and as mean $\pm \mathrm{SD}$ for continuous variables. Group comparisons with respect to categorical variables are performed using the Chi-square test. Comparisons of quantitative parameters was performed using one-way ANOVA. A probability value of $P<0.05$ was considered statistically significant.

\section{Results}

During the study, all asthma patients were divided into 2 groups. Group 1 included 38 patients with BA; Group 2 included 65 patients with BA and OA. The formed groups did not differ by age, gender, marriage status, or level of education $(P<0.05)$.
BMI values were $26.76 \pm 2.09 \mathrm{~kg} / \mathrm{m}^{2}$ in Group 1 and $27.46 \pm 1.33 \mathrm{~kg} / \mathrm{m}^{2}$ in Group $2(P=0.04)$. There was no statistically significant difference in spirometry parameters between the groups. Laboratory test results are presented in the Table 1. The levels of leptin, TNF- $\alpha$, and Il-6 were significantly higher in Group 2 than in Group 1. On the contrary, the IL-4 level was higher in Group 1 than in Group 2 . The TAS value was significantly higher in Group 1 than in Group $2(P=0.0001)$. The TOD value was significantly higher in Group 2 than in Group $1(P=0.0000)$.

Table 1.

Biochemical parameters in the study groups

\begin{tabular}{|l|c|c|c|}
\hline \multicolumn{1}{|c|}{ Variable } & Group 1 & Group 2 & $P$-value \\
\hline Leptin, $\mathrm{ng} / \mathrm{ml}$ & $25.11 \pm 5.44$ & $27.83 \pm 4.77$ & 0.0093 \\
\hline Adiponectin, $\mu \mathrm{g} / \mathrm{ml}$ & $11.22 \pm 4.85$ & $9.82 \pm 2.30$ & 0.0502 \\
\hline IL-6, $\mathrm{pg} / \mathrm{ml}$ & $16.79 \pm 5.61$ & $19.77 \pm 4.89$ & 0.0057 \\
\hline IL-4, $\mathrm{pg} / \mathrm{ml}$ & $6.83 \pm 3.81$ & $5.05 \pm 2.41$ & 0.0045 \\
\hline TNF- $\alpha, \mathrm{pg} / \mathrm{ml}$ & $2.14 \pm 0.60$ & $2.63 \pm 0.61$ & 0.0000 \\
\hline TAS, $\mu \mathrm{mol} / 1$ & $304.62 \pm 80.48$ & $248.30 \pm 55.58$ & 0.0001 \\
\hline TOS, $\mu \mathrm{mol} / 1$ & $1443.16 \pm 691.74$ & $2146.99 \pm 537.40$ & 0.0000 \\
\hline
\end{tabular}

$\mathrm{AQLQ}(\mathrm{S})$ questionnaire results are presented in the Table 2. The domains of AQLQ(S) activity, symptoms, and emotions were decreased in patients of Group 2. The AQLQ(S) environment domain was also decreased in patients of Group 2: $3.67 \pm 1.13$ points compared with $4.04 \pm 1.53$ points in Group 1; however, the difference was not statistically significant.

The values of the ACT test were $18.0 \pm 2.61$ points and $16.78 \pm 1.92$ points in Group 1 and Group 2, respectively $(P=0.0077)$. AQLQ $(\mathrm{S})$ total score positively correlated with the ACT score $(\mathrm{r}=0,77 ; P<0,05)$, IL-4 level $(\mathrm{r}=0,53 ; P<0,05)$, and TAS level $(\mathrm{r}=0,53 ; P<0,05)$, and had an inverse correlation with the levels of IL-6(r=-0,67; $P<0,05)$, TOS $(\mathrm{r}=-0,68 ; P<0,05)$, and TNF- $\alpha(\mathrm{r}=-0,47 ; P<0,05)$. The Il-6 level had a positive correlation with BMI $(\mathrm{r}=0,82 ; P<0,05)$, leptin level $(\mathrm{r}=0,83$; $P<0,05)$, TNF- $\alpha$ level $(\mathrm{r}=0,70 ; \mathrm{P}<0,05)$, and TOS $(\mathrm{r}=0,80$; $P<0,05)$, and an inverse correlation with adiponectin level $(\mathrm{r}=-$ $0,79 ; P<0,05)$ and TAS $(\mathrm{r}=-0,80 ; P<0,05)$.

Table 2.

$A Q L Q(S)$ questionnaire results

\begin{tabular}{|l|c|c|c|}
\hline \multicolumn{1}{|c|}{ Domain } & Group 1 & Group 2 & $P$-value \\
\hline Activity, points & $4.70 \pm 1.08$ & $3.46 \pm 0.56$ & 0.0000 \\
\hline Symptoms, points & $4.38 \pm 0.85$ & $3.34 \pm 0.53$ & 0.0000 \\
\hline Environment, points & $4.04 \pm 1.53$ & $3.67 \pm 1.13$ & 0.1635 \\
\hline Emotions, points & $4.68 \pm 1.10$ & $3.50 \pm 0.61$ & 0.0000 \\
\hline Total score, points & $4.69 \pm 1.11$ & $3.49 \pm 0.59$ & 0.0000 \\
\hline
\end{tabular}

\section{Discussion}

There is very limited data on the association between asthma and osteoarthritis. Koo et al. ${ }^{(10)}$ analyzed 9344 people, 
among whom the percentage of patients with asthma was $4.6 \% \pm 0.3 \%$. The prevalence of OA in the asthma group was $31.9 \pm 2.8 \%$, which was significantly higher than in the COPD group $(17.8 \% \pm 1.5 \%)$ or control group $(16.2 \% \pm 0.6 \%)$. OA was predominant in asthma patients after adjusting for age, sex, BMI, and smoking status (OR=1.65; 95\% CI: 1.27-2.13). After further adjusting this model for an OA drug, OA still remained independently associated with asthma(OR 1.56; 95\% CI 1.10-2.20). Moreover, radiographic severity of knee $\mathrm{OA}$ was correlated with asthma $(\mathrm{OR}=1.10 ; 95 \% \mathrm{CI}: 1.0$ 1.21. In another study on the association between BA and OA by Mahmood et al., ${ }^{(1)}$ the authors examined 56 patients with BA and OA over the age of 40 . They found that asthma patients suffered from more severe knee pain than the general population (control group) $(P=0.001)$. On the other hand, these asthmatic patients had the same frequency and severity of hip pain as the general population $(P=0.162)$. One of the most important findings was that functional impairment, as measured by the WOMAC scale, was very high among asthma patients with osteoarthritis. They also found that the rate of emergency hospitalization for asthmatic patients, which reflects the severity and uncontrollability of asthma, was associated with a higher risk of developing OA $(P=0.044)$. In our study, we had similar results - asthmatic patients with OA had worse asthma control than patients without OA.

Both BA and OA are associated with the IL-6 level. In asthmatic patients, serum levels of IL-6 were higher in allergic and non-allergic asthma than in healthy controls, and those levels increased in exacerbations; the IL-6 level also negatively correlated with ACT score, indicating an association with poor asthma control. ${ }^{(12,13)}$ Serum IL-6 is a significant predictor of radiographic OA. ${ }^{(14)}$ High IL-6 serum levels were also found in patients with symptomatic OA. ${ }^{(15)}$

In our study, patients with both BA and OA had higher levels of IL-6 than asthmatic patients without OA. The IL-6 level also had a negative correlation with ACT score and a positive correlation with leptin level. Leptin is a proinflammatory cytokine, which induces release of IL-6 and TNF- $\alpha .{ }^{(16,17)}$ High leptin levels are associated not only with knee and hip OA, but also with hand OA. ${ }^{(18)}$ Studies have shown that high leptin levels can also be found in synovial fluid, and synovial fibroblasts showed a dose-dependent increase in the expression of IL-6 when treated with leptin. ${ }^{(19,20)}$ This suggests that leptin can promote the progression of OA through IL-6. At the same time, leptin also affects lung cells, promoting allergic airway inflammation as it influences protein response factor XBP1s in an mTOR- and MAPK-dependent manner in proallergic TH2 cells. ${ }^{(21)} \mathrm{TNF}-\alpha$ is known to play an important role in OA pathogenesis. One of the reasons for that could be that chondrocytes in human OA cartilage have a high expression of the p55 TNF- $\alpha$ receptor, which could make OA cartilage particularly susceptible to TNF- $\alpha$ degradative stimuli. ${ }^{(22)} \mathrm{TNF}-\alpha$ also has been studied in asthma: high serum levels of TNF- $\alpha$ were found in asthmatic patients. ${ }^{(23)}$ In our study TNF- $\alpha$ levels were higher in patients with BA and OA.

Several studies showed that OA progression is related to oxidative stress. ${ }^{(24)}$ The most likely initial pathological syndrome in $\mathrm{OA}$ is the activation of the mitotic division of cartilage cells, with their production of proteoglycans, collagen II, and hypertrophy. The reason for these phenomena may be the predominance of oxidative damage over the antioxidant system. Oxidative stress has also been implicated in the pathology of asthma due to its induction of different proinflammatory mediators, enhancing bronchial hyperresponsiveness, stimulating bronchospasm, and increasing mucin secretion. ${ }^{(25)}$ In our study, oxidative stress was much more prominent in patients with BA and OA than in asthmatic patients without OA.

In conclusion, to our current knowledge there are no studies that concentrate on cytokine profile or quality of life in patients with asthma and osteoarthritis. Our study showed that in patients with both asthma and osteoarthritis, levels of inflammatory cytokines, such as leptin, IL- 6 , and TNF- $\alpha$, are significantly elevated as well as values of total oxidative status, which correlate with poorer asthma control and quality of life. Osteoarthritis is a severe co-morbid pathology in asthmatic patients. Further research on the association between asthma and osteoarthritis and its underlying mechanisms is required.

\section{Sources of Funding}

This work was supported by the Council on Grants of the President of the Russian Federation (NSh 4994.2018.7.).

\section{Competing Interests} interests.

The authors declare that they have no competing

\section{References}

1. Rehman A, Amin F, Sadeeqa S. Prevalence of asthma and its management: A review. J Pak Med Assoc. 2018 Dec;68(12):1823-1827.

2. McGeachie MJ, Wang AL, Lutz SM, Sordillo JE, Weiss ST, Tantisira KG, Iribarren C, Lu MX, Wu AC. Real-Life Patterns of Exacerbations While on Inhaled Corticosteroids and Long-Acting Beta Agonists for Asthma over 15 Years. J Clin Med. 2020 Mar 18;9(3):819. doi: 10.3390/jcm9030819.

3. Tsvetikova LN, Budnevsky AV, Ovsyannikov ES, Kudashova EA. Melatonin: Possibilities for use in the treatment of asthma. Ter Arkh. 2017;89(3):112-5. Russian. doi: 10.17116/terarkh2017893112-115.

4. Brussino L, Solidoro P, Rolla G. Is it severe asthma or asthma with severe comorbidities? J Asthma Allergy. 2017 Nov 29;10:303-305. doi: 10.2147/JAA.S150462.

5. Budnevsky A, Tribuntceva L, Kozhevnikova S, Ovsyannikov E. Impact of Metabolic Syndrome Components on Asthma Control and Life Quality of Patients. International Journal of Biomedicine. 2018;8(1):33-6. doi: 10.21103/ article8(1)_oa4.

*Corresponding author: Evgeniy S. Ovsyannikov, PhD, ScD. Department of Faculty Therapy, Voronezh State Medical University named after N.N. Burdenko. Voronezh, Russia. E-mail: ovses@ yandex.ru 
6. Budnevsky AV, Isaeva YV, Malysh EY, Kozhevnikova SA. [Pulmonary rehabilitation as an effective method for optimizing therapeutic and preventive measures in patients with chronic obstructive pulmonary disease concurrent with metabolic syndrome]. Ter Arkh. 2016;88(8):25-29. Russian. doi: 10.17116/terarkh201688825-29.

7. Budnevsky AV, Malysh EY, Ovsyannikov ES, Drobysheva ES. [Asthma and metabolic syndrome: Clinical and pathogenetic relationships]. Ter Arkh. 2015;87(10):110-114. doi: 10.17116/ terarkh20158710110-114. [Article in Russian].

8. Ermolova AV, Budnevsky AV, Yu ME, Ovsyannikov ES, Drobysheva ES. [BRONCHIAL ASTHMA AND METABOLIC SYNDROME]. Klin Med (Mosk). 2015;93(6):44-9. [Article in Russian].

9. Steppuhn H, Langen U, Keil T, Scheidt-Nave C. Chronic disease co-morbidity of asthma and unscheduled asthma care among adults: results of the national telephone health interview survey German Health Update (GEDA) 2009 and 2010. Prim Care Respir J. 2014 Mar;23(1):22-9. doi: 10.4104/ pcrj.2013.00107.

10. Koo HK, Song P, Lee JH. Novel association between asthma and osteoarthritis: a nationwide health and nutrition examination survey. BMC Pulm Med. 2021 Feb 16;21(1):59. doi: 10.1186/s12890-021-01425-6.

11. Mahmood ZA, Malghooth ZT. Relationship of Hips and Knees Osteoarthritis with Bronchial Asthma. Res J Pharm Biol Chem Sci. 2019; 10(2):64-70.

12. Ghebre MA, Pang PH, Desai D, Hargadon B, Newby C, Woods J, et al. Severe exacerbations in moderate-to-severe asthmatics are associated with increased pro-inflammatory and type 1 mediators in sputum and serum. BMC Pulm Med. 2019 Aug 8;19(1):144. doi: 10.1186/s12890-019-0906-7.

13. Dimitrova D, Youroukova V, Ivanova-Todorova E, Tumangelova-Yuzeir K, Velikova T. Serum levels of IL-5, IL-6, IL-8, IL-13 and IL-17A in pre-defined groups of adult patients with moderate and severe bronchial asthma. Respir Med. 2019 Jul-Aug;154:144-154. doi: 10.1016/j.rmed.2019.06.024.

14. Livshits G, Zhai G, Hart DJ, Kato BS, Wang H, Williams FM, Spector TD. Interleukin-6 is a significant predictor of radiographic knee osteoarthritis: The Chingford Study. Arthritis Rheum. 2009 Jul;60(7):2037-45. doi: 10.1002/ art.24598.

15. Imamura M, Ezquerro F, Marcon Alfieri F, Vilas Boas L, Tozetto-Mendoza TR, Chen J, Özçakar L, Arendt-Nielsen
L, Rizzo Battistella L. Serum levels of proinflammatory cytokines in painful knee osteoarthritis and sensitization. Int J Inflam. 2015;2015:329792. doi: 10.1155/2015/329792.

16. Matsusaka M, Fukunaga K, Kabata H, Izuhara K, Asano K, Betsuyaku T. Subphenotypes of type 2 severe asthma in adults. J Allergy Clin Immunol Pract. 2018 Jan-Feb;6(1):274-276.e2. doi: 10.1016/j.jaip.2017.06.015.

17. Provotorov VM, Budnevskii AV, Semenkova GG, Shishkina ES. [PROINFLAMMATORY CYTOKINES IN COMBINATION OF CORONARY HEART DISEASE AND CHRONIC OBSTRUCTIVE PULMONARY DISEASE]. Klin Med (Mosk). 2015;93(2):5-9. [Article in Russian]. 18. Morales Abaunza RA, Rojas ÁP, Rojas C, Motta O, Atuesta J, Alzate JP, et al. Levels of serum leptin in patients with primary hand osteoarthritis. Rev Colomb Reumatol. 2020;27:20-25. doi: 10.1016/j.rcreue.2019.12.005.

19. Yan M, Zhang J, Yang H, Sun Y. The role of leptin in osteoarthritis. Medicine (Baltimore). 2018 Apr;97(14):e0257. doi: 10.1097/MD.0000000000010257.

20. Budnevsky AV, Ovsyannikov ES, Labzhania NB. [Chronic obstructive pulmonary disease concurrent with metabolic syndrome: Pathophysiological and clinical features]. Ter Arkh. 2017;89(1):123-127. doi: 10.17116/ terarkh2017891123-127. [Article in Russian].

21. Zheng H, Wu D, Wu X, Zhang X, Zhou Q, Luo Y, Yang X, Chock CJ, Liu M, Yang XO. Leptin Promotes Allergic Airway Inflammation through Targeting the Unfolded Protein Response Pathway. Sci Rep. 2018 Jun 11;8(1):8905. doi: 10.1038/s41598-018-27278-4.

22. Fernandes JC, Martel-Pelletier J, Pelletier JP. The role of cytokines in osteoarthritis pathophysiology. Biorheology. 2002;39(1-2):237-46.

23. Ren J, Sun Y, Li G, Zhu XJ, Cui JG. Tumor necrosis factor- $\alpha$, interleukin- 8 and eosinophil cationic protein as serum markers of glucocorticoid efficacy in the treatment of bronchial asthma. Respir Physiol Neurobiol. 2018 Dec;258:8690. doi: 10.1016/j.resp.2018.06.004.

24. Zahan OM, Serban O, Gherman C, Fodor D. The evaluation of oxidative stress in osteoarthritis. Med Pharm Rep. 2020 Jan;93(1):12-22. doi: 10.15386/mpr-1422.

25. Provotorov VM, Budnevsky AV, Filatova YI, Perfil'eva MV. [ANTIOXIDANT THERAPY OF BRONCHIAL ASTHMA]. Klin Med (Mosk). 2015;93(8):19-22. [Article in Russian]. 